\title{
UCS intensity-dependent attenuation of infantile amnesia
}

\author{
ROBERT W. FLINT, JR. \\ The College of Saint Rose, Albany, New York \\ and \\ MICHAEL D. BUNSEY and DAVID C. RICCIO \\ Kent State University, Kent, Ohio
}

\begin{abstract}
Unconditioned stimulus (UCS) reactivation treatments may attenuate infantile amnesia in rats by providing an external reminder cue, creating an internal contextual reminder cue via endogenous hormones, and/or modulating memory retrieval via neurochemical changes from activation of the stress cascade. In the present study, the UCS reactivation treatment and the relationship between intensity at training and intensity at testing were examined. In Experiment 1, 17-day-old Sprague-Dawley rats were given multiple-trial passive-avoidance conditioning with a high-intensity footshock. Those subjects administered a high-intensity reactivation treatment performed significantly better than did subjects that received a low-intensity reactivation treatment, subjects that received no treatment, or pseudo-trained animals. Experiment 2 repeated the first experiment with a low-intensity training footshock. The results indicated that only a high-intensity reactivation treatment attenuated infantile amnesia. These findings suggest that the training UCS need not match the reactivation treatment in terms of intensity and leaves open the possibility that aversive reactivation treatments may be multifaceted, involving external contextual cues, internal contextual cues, and neuromodulatory effects from activation of the stress cascade.
\end{abstract}

In the first ontogenetic experiment on long-term memory in rats, Campbell and Campbell (1962) found that retention varied as a function of age. Animals that were 18 days of age when trained showed a disruption in performance at a 7 -day delay and little retention at all 21 days following training. Adults ( 100 days of age), on the other hand, had excellent retention for up to $\mathbf{4 2}$ days following training. Such retention deficits in infant and juvenile rats have also been demonstrated by using an appetitive discrimination task (Campbell, Jaynes, \& Misanin, 1968) and an active avoidance task (Kirby, 1963). Similar retention deficits have been demonstrated in immature humans (Rovee-Collier \& Hayne, 1987; Rovee-Collier \& Shyi, 1992; Usher \& Neisser, 1993), and this phenomenon is now generally referred to as infantile amnesia (IA).

Most research on IA has focused on either its causes or the characteristics of its development and alleviation. In two comprehensive reviews, Campbell and Spear (1972) and Howe and Courage (1993) presented many potential

The studies reported here were previously presented at the 71 st annual Midwestern Psychological Association meeting, Chicago. This research was supported, in part, by National Institute of Mental Health Grant MN37535 to D.C.R. These studies were completed in partial fulfillment of the requirements for the first author's doctoral degree. Correspondence concerning this article should be addressed to $\mathrm{R}$. W. Flint, Jr., The College of Saint Rose, Department of Psychology, 432 Western Avenue, Albany, NY 12203-1490 (e-mail: flintr@mail.strose.edu). explanations for this unusual phenomenon, including maturational changes in interference and informationprocessing mechanisms, changes in the structure and chemical make-up of the central nervous system during development, changes in perception during the retention interval, and, in humans, the personalization of event memory and the development of a cognitive sense of self. Currently, little agreement exists as to the cause of this phenomenon, although researchers generally agree now that IA represents a retrieval failure at the time of testing, as opposed to a failure to store information at the time of acquisition.

Other laboratories have focused on treatments that modulate the animal's performance at the time of the retention test. The vast majority of studies demonstrating the alleviation of the retrieval deficits associated with IA comes from two separate research paradigms, reinstatement and reactivation (Spear \& Riccio, 1994). In the reinstatement paradigm, multiple reminder treatments are administered between the training episode and the retention test. This procedure may be contrasted with the reactivation paradigm in which the reminder treatment is only administered once between training and testing, and then, typically, at least $24 \mathrm{~h}$ prior to the retention test.

In a demonstration of reinstatement, Campbell and Jaynes (1966) conditioned fear in 25-day-old weanling rats. On Days 7, 14, and 21 following conditioning, these subjects received a single reinstatement treatment (a footshock) consisting of reexposure to the training uncondi- 
tioned stimulus (UCS) in the context in which conditioning originally occurred. Fifty-three days after the original conditioning (i.e., as adults), these subjects were tested for conditioned fear and displayed significantly higher retention scores than did conditioned subjects that had not received the reinstatement treatments and subjects that had received the reinstatement treatments but had not received the original conditioning. These findings suggest that periodic reexposure to the training reinforcer is sufficient to reinstate and maintain the memory in an accessible state, allowing subjects to retrieve the learning episode when presented with the training context.

Spear and Parsons (1976) examined the reinstatement effect and compared it with a reactivation paradigm. In these experiments, the most effective reinstatement and reactivation treatment involved exposure to the conditioning UCS (footshock). They demonstrated that a UCS reactivation treatment $24 \mathrm{~h}$ prior to testing had differential effects on retention performance that were based on the age at which training occurred. Rats were trained at 16 days, 23 days, or as adults and were tested 28 days later. The reactivation treatment was effective for all groups, with the exception of the 16-day-old subjects. However, Spear and Parsons did find that the reactivation treatment was effective for subjects at this age if the retention interval was shortened to 7 days instead of 28 . These results suggest that the training memory was stored but that it is likely that the effectiveness of the reactivation treatment decreases as the retention interval increases. Furthermore, a single exposure to the conditioning reinforcer is sufficient to reactivate the training memory, rather than multiple exposures as in the reinstatement paradigm. The results from these studies show that younger subjects forget more rapidly than older subjects, which coincides with the data from studies of human infants.

The effectiveness of an aversive and stressful reactivation treatment such as footshock may be multidimensional. First, as an external cue, it is a distinct, acute, and mildly painful stimulus that is novel to the organism at conditioning and, thus, serves as a cue associated only with training. Second, footshock induces psychological states, such as fear and emotional arousal, in the animal, which may serve to produce some sort of internal contextual cue. Finally, most significant stressors activate the stress cascade, which leads to a neuroendocrine response, including activation of the sympathetic nervous system and the hypothalamic-pituitary-adrenal axis. This activation induces the release of ACTH from the anterior pituitary, glucocorticoids from the adrenal cortex, epinephrine from the adrenal medulla, and glucose from the liver. Incidentally, all of these neurochemicals have been shown to modulate mnemonic processes in adult rodents (Gold, 1995; Gold \& van Buskirk, 1975, 1976; Haroutunian \& Riccio, 1977, 1979; Roozendaal, Williams, \& McGaugh, 1999; Sandi, 1998; White, 1991; White \& Legree, 1984).

In virtually all studies of IA that use the reactivation paradigm, the treatment is administered at least $24 \mathrm{~h}$ prior to the retention test, in order to avoid any behavioral side effects that might alter the subject's behavior during the test. Although this procedure is followed for a good reason (to avoid nonmemorial treatment effects on behavior during retention tests), it may be beneficial to use shorter intervals to understand further why certain treatments are effective and others are not.

In a recent exception to the typical reactivation paradigm, Miller, Jagielo, and Spear (1991) presented reactivation treatments shortly prior to the retention test. In their study, 18-day-old animals were trained in an odor $\mathrm{CS}-/ \mathrm{CS}+$ conditioning paradigm where the CS+ was paired with a footshock and the CS- was safe. Five minutes prior to the retention test, either 3 or $24 \mathrm{~h}$ after conditioning, subjects were administered a 30-sec exposure to the UCS, the CS+, the CS-, the context, or a novel odor as a reactivation treatment. Miller et al. demonstrated that the UCS, the CS - , and the CS+ were effective reminder treatments at both the 3-and the 24-h retention intervals and that a reactivation treatment administered as soon as $5 \mathrm{~min}$ prior to the retention test can attenuate retrieval deficits (unpaired controls demonstrated that the reactivation treatments did not have any behavioral effects that might be construed as retention). These findings indicate that reactivation treatments may be effectively administered almost immediately prior to the retention test and that, with the appropriate controls, memory retrieval may be examined. This technique of administering reactivation treatments shortly prior to the retention test may allow researchers to systematically examine the effects of various treatments on retrieval.

The following experiments were developed to examine the UCS (footshock) reactivation effect by using a reactivation paradigm for passive-avoidance conditioning in preweanling rats. In Experiment 1, the UCS as a reactivation treatment was investigated by comparing low-and high-intensity footshocks in animals that had been given passive-avoidance conditioning with a high-intensity UCS. Although the UCS has been highly effective in reactivating memory, it has always been administered for the same duration and at the same intensity as those during conditioning. In Experiment 2, the UCS reactivation effect was examined by using a low-intensity footshock as the training stimulus and then administering either the low- or the high-intensity UCS as a reactivation treatment in the same manner as that in Experiment 1. If a distinct match between the external and internal contextual cues and the neurochemical responses are necessary for memory reactivation, the high-intensity reactivation treatment should be the more effective in Experiment 1, whereas the low-intensity reactivation treatment should be more effective in Experiment 2.

\section{EXPERIMENT 1}

Prior reactivation studies have administered the reactivation treatment at least $24 \mathrm{~h}$ prior to the retention test. 
In those studies using footshock as the reactivation treatment, it was typically administered for the same duration and at the same intensity as those during training. In the present experiment, the effectiveness of either a low- or a high-intensity reactivation treatment administered shortly prior to testing in preweanling rat pups that had been previously given passive-avoidance conditioning with a high-intensity UCS was assessed. The question of whether it is necessary for the reactivation treatment to match the training UCS was also examined.

\section{Method}

Subjects. Thirty-five male naive 17-day-old Sprague-Dawley rat pups and 7 male 90-day-old adult Sprague-Dawley rats served as subjects. The animals were obtained from Hilltop Lab Animals (Scottsdale, PA), and the age of the animals was determined by counting date of birth as Day 0 . All the pups were maintained at the Kent State University vivarium in litters of 10 subjects each in Plexiglas cages on a 15:9-h light:dark cycle. One dam was also included in each cage, with food and water available ad lib. The animals were purchased with special instructions so that no two animals within a single litter were from the same dam. This precaution, in addition to selecting no more than 2 subjects from each litter for group assignment, was used to help reduce any intralitter correlations (see Holson \& Pearce, 1992, for discussion of issues associated with sampling in multiparous species). The adults were maintained in hanging wire mesh cages in groups of 3 or 4 animals on a 15:9-h light:dark cycle, with food and water available ad lib. At the time of training, the weights for the infants ranged from 25 to $51 \mathrm{~g}$, whereas the adults ranged from 360 to $385 \mathrm{~g}$.

Apparatus. Standard black-and-white passive-avoidance boxes were used for training and testing. The main chamber of the infant apparatus measured $40 \times 18 \times 11 \mathrm{~cm}$ and was constructed of Plexiglas and divided into two equal-sized compartments separated by a guillotine door $(11 \times 11 \mathrm{~cm})$. One compartment was made from white translucent Plexiglas with a clear lid. The other compartment was made entirely from black opaque Plexiglas. The color of the guillotine door dividing the chambers was consistent with the color of the compartment it faced. The adult apparatus was the same as the one used for the infants, with the exception that it was of slightly different dimensions, to account for the size of the animals. The adult apparatus measured $33 \times 17 \times 17 \mathrm{~cm}$, with a guillotine door measuring $10 \times 8 \mathrm{~cm}$. The floors of both chambers were constructed of stainless steel bars approximately $2 \mathrm{~mm}$ in diameter and spaced $1 \mathrm{~cm}$ apart. A $1-\mathrm{sec} 1.38-\mathrm{mA}$ unscrambled shock from a matched impedance ac shock source could be administered through these bars to the animals by the experimenter. The room where training and testing occurred was illuminated with a 15-W light bulb suspended $30 \mathrm{~cm}$ above the white compartment and was equipped with a white noise generator to mask any background disturbances.

Noncontingent footshocks for training and memory reactivation prior to testing were administered in a different room and in a different apparatus. The apparatus was a rectangular box constructed from plywood, with dimensions measuring $21 \times 18 \times 19 \mathrm{~cm}$ and with a clear Plexiglas lid. The floor consisted of stainless steel bars $2 \mathrm{~mm}$ in diameter and spaced $1 \mathrm{~cm}$ apart. A $1-\mathrm{sec}$ footshock could be delivered by the experimenter through the grid floor from a matched impedance ac shock source. The room was illuminated with overhead fluorescent lighting, and treatments were administered in the absence of a white noise generator. A hand-held stopwatch was used to measure the latency and total time on white dependent variables. The same experimenter conducted the training, reactivation, and testing phases of the experiment.

Procedure. No more than 2 infants from each litter were randomly assigned to one of five groups: immediate (IMM, $n=7$ ), pseudo-training (PSEUDO, $n=6$ following the death of 1 subject during the delay between training and testing), trained control (CTRL, $n=7$ ), low-intensity footshock reactivation treatment (UCS-.40, $n=7$ ), and high-intensity footshock reactivation treatment (UCS-1.38, $n=7$ ).

The animals in the adult group $(n=7)$ received multiple-trial passive-avoidance conditioning and were returned to their home cages before being given a retention test $24 \mathrm{~h}$ later, as will be described below. The IMM group of preweanling rats received multiple-trial passive-avoidance conditioning. Following training, these animals were placed individually into small clear Plexiglas cages for 5 min before being tested for retention. Infants in the CTRL group were given multiple-trial passive-avoidance conditioning and returned to the litter. Twenty-four $h$ later, they were placed individually into the noncontingent footshock apparatus for $30 \mathrm{sec}$ (no shock was administered) in order to control for handling, intertrial interval, and exposure to the apparatus. Twenty-five $( \pm 5)$ min later, these were tested for retention of the passive-avoidance conditioning. The animals in the UCS-.40 and UCS-1.38 reactivation groups received multiple-trial passive-avoidance conditioning and were returned to their litters. Twenty-four $h$ later, these animals were placed individually into the noncontingent footshock apparatus, where, after a 10 sec delay, the UCS-.40 group received a $1-\mathrm{sec} 0.40-\mathrm{mA}$ footshock and the UCS-1.38 group received a $1-\sec 1.38-\mathrm{mA}$ footshock before being placed individually into small Plexiglas cages. Approximately $25( \pm 5) \mathrm{min}$ later, animals were tested for retention of the passive-avoidance conditioning. Finally, preweanling rats in the PSEUDO group were placed individually into the noncontingent footshock apparatus, where they received three $1-\mathrm{sec} 1.38-\mathrm{mA}$ footshocks, each separated by a 10-sec delay. The number of pseudotraining trials administered to the PSEUDO group was based on the average number of trials to criterion for the other preweanling groups. Approximately $24 \mathrm{~h}$ after pseudo-training, the animals were given a 1-sec 1.38-mA reactivation noncontingent footshock, were placed individually into a small clear Plexiglas cage for $25( \pm 5) \mathrm{min}$, and were then tested in the passive-avoidance apparatus. The PSEUDO group was included in order to determine whether exposure to footshock, in the same pattern as preweanling rat pups receiving passive-avoidance conditioning and reactivation treatment, would alter the behavior of the subjects regardless of exposure to the actual passive-avoidance apparatus.

All the subjects receiving passive-avoidance conditioning were brought into the lab and allowed to view their surroundings for $10 \mathrm{sec}$ from the experimenter's hand. The subjects were then placed into the white (safe) compartment of the apparatus facing away from the guillotine door. After $10 \mathrm{sec}$, the door was raised, and each animal's latency to cross over into the black compartment (four-paw criterion) was recorded as a dependent measure. If the subjects did not cross over on the initial trial of training in $60 \mathrm{sec}$, they were removed from the experiment. After crossing into the black compartment, the guillotine door was closed, and a 1-sec 1.38-mA footshock was immediately administered. At the cessation of the footshock, the subject was removed and returned to the white compartment, and the procedure was repeated until the subject did not cross over to the black chamber for 180 consecutive sec. The number of trials to criterion was also recorded as an additional dependent measure.

Reactivation treatments were administered $25( \pm 5)$ min prior to testing and consisted of a $1-\mathrm{sec}$ footshock of either a low intensity $(0.40 \mathrm{~mA})$ or a high intensity $(1.38 \mathrm{~mA})$. Prior research had established that more than $90 \%$ of the animals of this strain and age vocalized when administered a 1-sec 0.37-mA footshock (Flint, Bunsey, \& Riccio, 1997b). On the basis of these data, a $0.40-\mathrm{mA}$ footshock was selected as the low-intensity footshock reactivation treatment.

For the retention test, the subjects were brought into the test room, held for $10 \mathrm{sec}$ by the experimenter, and then placed on the white side of the apparatus, facing away from the guillotine door. After 
25 -

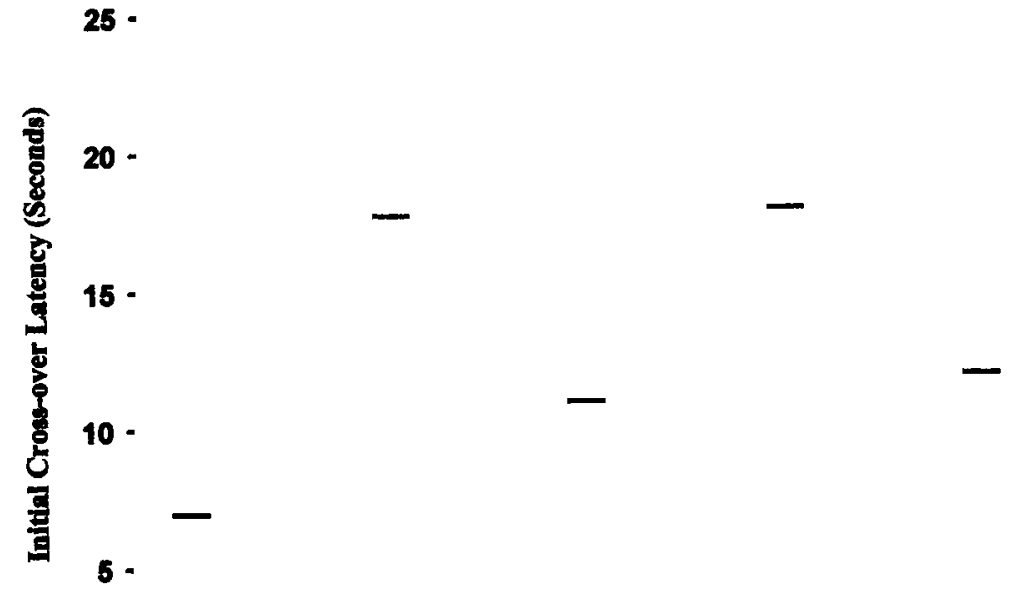

$\mathbf{0}$
Adult
IMM
CTRL
UCS-40
UCS-1.38

Figure 1. Mean initial crossover latencies from the first trial of training for the immediate test (IMM), control (CTRL), low UCS reactivation treatment (UCS-.40), and high UCS reactivation treatment (UCS-1.38) groups of adults. Error bars represent standard errors of the mean.

$10 \mathrm{sec}$, the guillotine door was raised, and the subject's initial crossover latency and total time spent on the white side (TTW) during the 5-min retention test were recorded as dependent measures of fear retention.

\section{Results and Discussion}

The statistical analysis of the data involved parametric tests whenever possible, but in circumstances in which the data did not meet the necessary assumptions, nonparametric tests were employed.

The mean latencies for the first training trial and the mean number of trials to criterion during training for each group are displayed in Figures 1 and 2, respectively. These figures suggest that the adult group may have had more rapid crossover latencies on the initial training trial and that they may have required fewer trials to criterion than did the preweanling groups.

A one-way analysis of variance (ANOVA) on the initial crossover latencies from training for all of the groups, except the PSEUDO group, indicated that there were no differences among these groups at the time of training $[F(4,30)=1.89, p>.10]$. A Kruskal-Wallis one-way ANOVA on the number of trials to criterion during training yielded a marginally significant effect $(H=9.24, p=$ .06 ); however, when the adult group was dropped from the analysis, the subsequent ANOVA was insignificant $(H=$ $0.94, p>.82$ ). These results suggest that the infants were equivalent at the time of training. The adults, although having equivalent training crossover latencies, required fewer trials to reach criterion than did the infants. This is consistent with other reports demonstrating an improve- ment in acquisition as age increases (Riccio, Rohrbaugh, \& Hodges, 1968).

The mean test latencies and TTW scores are shown in Figure 3, panels A and B, respectively. Both dependent measures clearly demonstrate the high level of retention by the adult and IMM groups. However, the CTRL group demonstrated very poor retention, as indicated by the low latency and TTW scores. This impression, in conjunction with the excellent performance of the adult and IMM groups, suggests that the retention loss in the CTRL group reflected IA. The UCS-1.38 group also displayed good retention, whereas the UCS-.40 and PSEUDO groups all performed very poorly. The good performance of the UCS-1.38 group would seem to indicate that the highintensity reactivation treatment was the most effective reactivation treatment administered, and because the PSEUDO group also performed poorly, the memory reactivation effect seen in the UCS-1.38 group cannot be explained by behavioral side effects of the treatment.

Analysis of the test latencies and TTW scores, using the Kruskal-Wallis one-way ANOVA, indicated that significant differences were present among the groups on both the latency and the TTW dependent measures $(H=21.87$ and $H=27.60$, respectively, $p s<.001$ ). All subsequent pairwise comparisons were conducted by using the twotailed Mann-Whitney $U$ test.

The pairwise comparisons confirmed the impressions based on the figures. Both the adult and the IMM groups had significantly longer latency $(U=0.00$ and $U=3.00$, respectively, $p \mathrm{~s}<.01)$ scores when compared with the CTRL group, indicating that IA had occurred. A compar- 


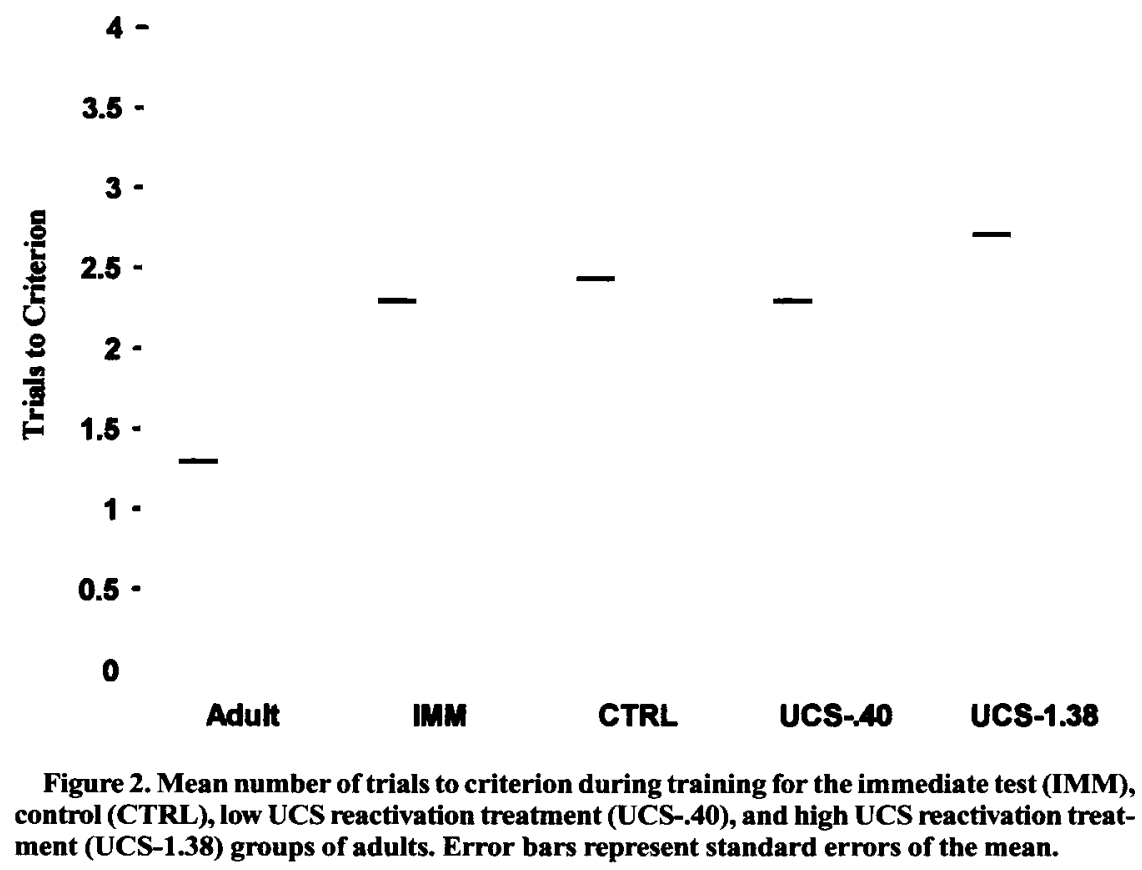

ison between the PSEUDO and the CTRL groups indicated that the high-intensity footshock used for memory reactivation did not influence behavior in a way that might be erroneously construed as retention (latency, $U=18.00$, and TTW, $U=9.00, p s>.05$ ).

When compared with controls, the UCS-. 40 group did not show any significant enhancement in memory retrieval, as is indicated by latency and TTW measures ( $U=20.00$ and $U=16.00$, respectively, $p \mathrm{~s}>.05$ ). However, the UCS-1.38 group did differ significantly from the CTRL group (latency, $U=8.00$, and TTW, $U=6.00, p s<$ .05 ), indicating that the high-intensity footshock reactivated the training memory and resulted in longer retention scores. Comparisons between the PSEUDO group and the UCS-1.38 group indicated that there were significantly longer retention scores for both dependent measures (latency, $U=3.00$, and TTW, $U=2.00, p s<.01$ ). These findings provide additional evidence for the memory reactivating effects of a high-intensity footshock.

These findings replicated prior studies employing a reactivation UCS treatment equivalent to the training stimulus. The finding that the low-intensity UCS was ineffective and the high-intensity reactivation treatment was effective suggests that footshocks of differing intensities may be perceived as different stimuli by these preweanling rats. This may explain the poor performance of the UCS-.40 group, since it did not match the intensity of the training UCS. The high-intensity reactivation treatment may have created an internal hormonal cue and/or activated the neuroendocrine components involved in memory modulation, but it is feasible in the present experiment to expect a difference between the reactivation treatments based solely on the difference in external contextual cues produced by the UCS.

\section{EXPERIMENT 2}

This experiment was designed to further investigate the characteristics of the UCS reactivation treatment on memory retrieval. In Experiment 1, the animals were trained with a high-intensity footshock and then were given reactivation treatments, using either a footshock of the same intensity or one that did not match the training stimulus in terms of intensity. The findings in that experiment suggested that the high-intensity reactivation treatment could have had its effect as a result of a match in the external cues provided by the stimulus or as a result of some other internal or, possibly, neurochemical alteration.

The purpose of this experiment is to examine the possible alternative that a match between the training and the reactivation stimulus accounts for the results in Experiment 1 . If a match in the external stimulus properties is necessary, animals trained with a low-intensity footshock should also display enhanced retention scores when administered a reactivation treatment of the same intensity prior to testing.

\section{Method}

Subjects. Sixty male naive 17-day-old Sprague-Dawley rat pups served as subjects. All animals were purchased from Hilltop Lab Animals (Scottsdale, PA) and maintained in litters of 10 subjects each under the same conditions as those outlined in Experiment 1. Specifically, no 2 pups in the same litter came from the same dam, and the age of the animals was determined by treating the date of birth as Day 0 . The weights of the preweanling pups at the time of training ranged from 34 to $46 \mathrm{~g}$.

Apparatus. The same passive-avoidance apparatus and noncontingent footshock (pseudo-training) apparatus as those used in Experiment 1 were used for training, testing, and reactivation treatments in the present study. Latencies for training and testing, as well as TTW scores during testing, were recorded with a hand-held 


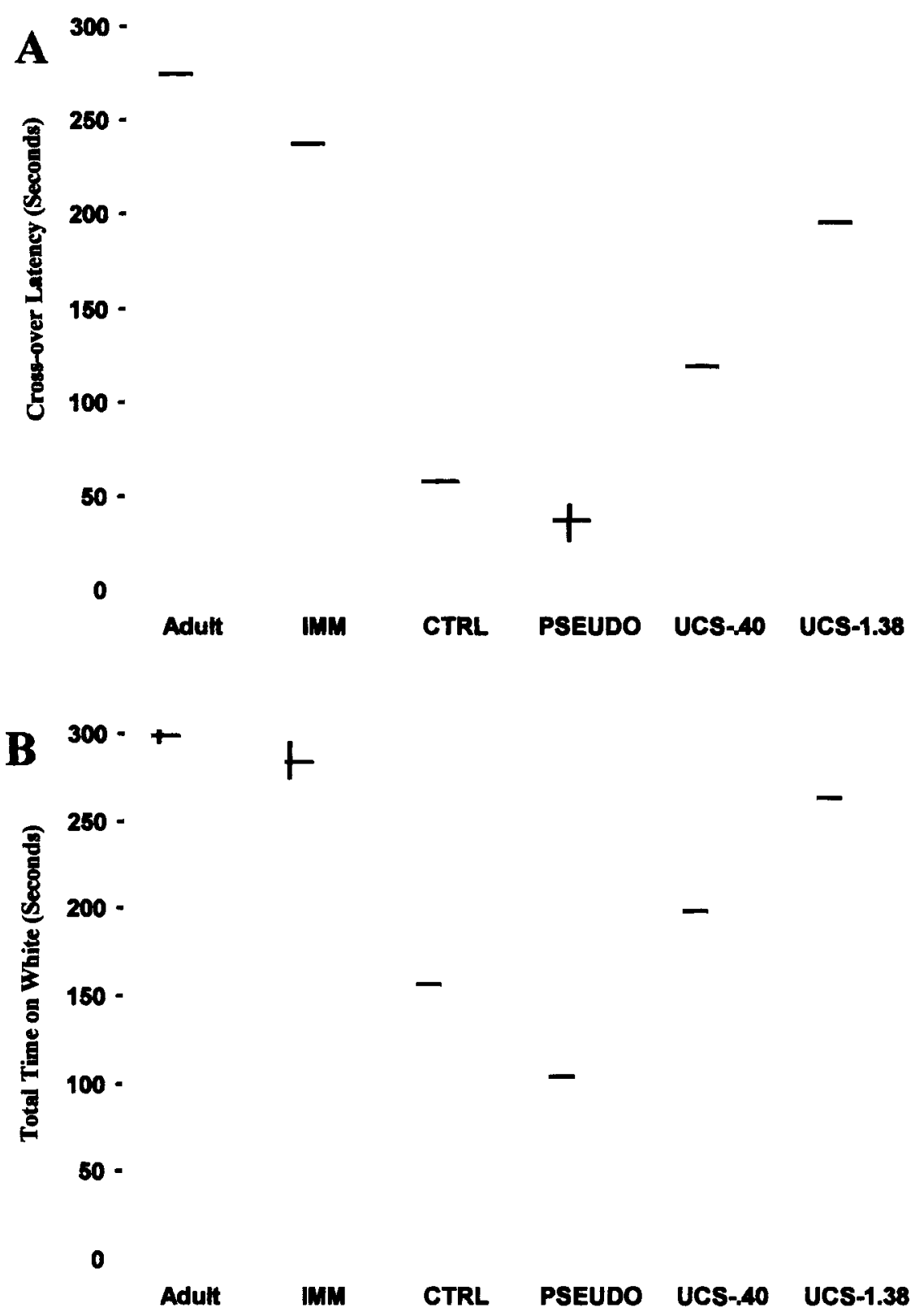

Figure 3. Mean crossover latencies (panel $A$ ) and scores for total time spent on white side (panel B) during testing for the immediate test (IMM), control (CTRL), pseudo-training (PSEUDO), low UCS reactivation treatment (UCS-.40), and high UCS reactivation treatment (UCS-1.38) groups of adults. Error bars represent standard errors of the mean.

stopwatch. The same researcher conducted the training, testing, and reactivation phases of the experiment.

Procedure. The animals from each litter were randomly assigned to each of six groups $(n \mathrm{~s}=10)$, with no more than 2 subjects from a single litter assigned to any one group. The same experimental design and procedure as those used for training and testing preweanling rat pups in Experiment 1 were used in the present experiment, yielding CTRL, IMM, UCS-.40, and UCS-1.38 groups, and a pseudo-training group reactivated with a $1.38-\mathrm{mA}$ footshock (PSEUDO-1.38). An additional pseudo-training group was in- cluded that was given a $0.40-\mathrm{mA}$ reactivation footshock (PSEUDO.40). The animals in the PSEUDO groups were trained with noncontingent footshocks, as in Experiment 1, but received a total of four 1 -sec $0.40-\mathrm{mA}$ footshocks, each separated by $10 \mathrm{sec}$. The number of footshocks was based on the average number required for training in the CTRL, IMM, UCS-.40, and UCS-1.38 groups. No adult animals were included in the present experiment.

The same passive-avoidance conditioning, pseudo-conditioning, and reactivation procedures as those outlined in Experiment 1 were used here, with the exception that the animals were trained with a 


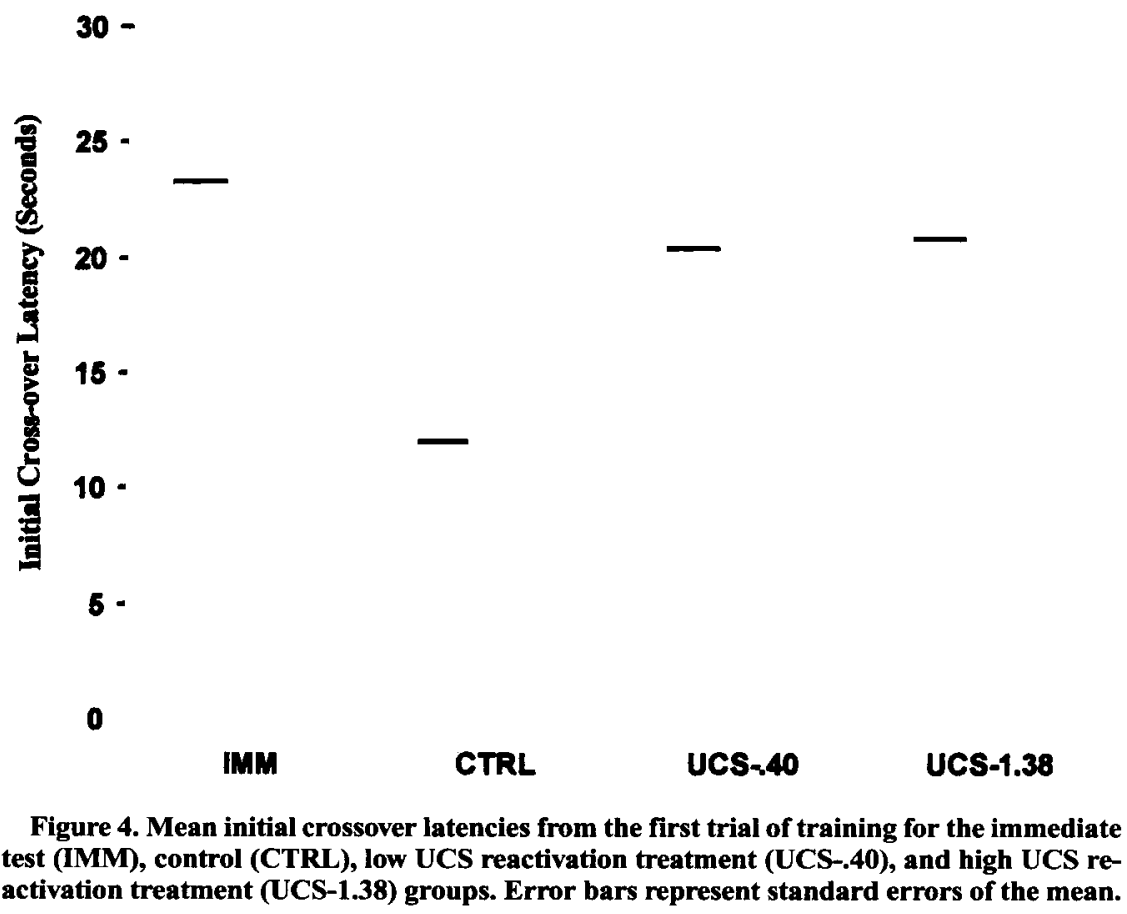

1-sec $0.40-\mathrm{mA}$ footshock, as opposed to the $1.38-\mathrm{mA}$ footshock used for training in Experiment 1. During the initial trial of passiveavoidance conditioning for the CTRL, IMM, UCS-.40, and UCS1.38 groups, a total of 10 subjects did not cross over within $60 \mathrm{sec}$, the established criterion for the initial training trial. The removal of these subjects from the experiment reduced the number of subjects in each of these groups (CTRL, $n=7$; IMM, $n=7$; UCS-.40, $n=8$; UCS-1.38, $n=8$ ).

\section{Results and Discussion}

Parametric statistical analyses were used whenever possible, but when the data did not meet the necessary assumptions, nonparametric tests were employed.

The mean latencies for the first training trial and the mean number of trials to criterion for each group are displayed in Figures 4 and 5, respectively. These figures suggest that the animals in the CTRL group may have had more rapid crossover latencies than did the subjects in other groups, but there are no apparent differences in the number of trials to criterion during training.

A one-way ANOVA on the initial crossover latencies for groups that received passive-avoidance conditioning indicated that there were no differences among these groups at the time of training $[F(3,26)=0.71, p>.10]$. A Kruskal-Wallis one-way ANOVA for the number of trials to criterion during training also indicated that there were no differences among the groups $(H=0.90, p>$ .10). These results clearly indicate that, at the time of training, there were no differences among the groups that received passive-avoidance conditioning.

The mean test latency and TTW scores for each group are shown in Figure 6, panels A and B, respectively. The high scores in the IMM group on both dependent measures suggest that the animals had good retention of the multiple-trial passive-avoidance conditioning for at least 5 min following training. In comparison with the IMM group, the CTRL group had very poor retention, as indicated by low latency scores. The UCS-1.38 group also appears to have better retention, in comparison with the CTRL group. There are no other obvious impressions from the latency data, but the TTW scores in Figure 6 appear to exhibit more substantial differences between the groups. This result is not surprising, considering that prior studies have reported that TTW scores are more sensitive than are latency scores in adult rodents (Flint, Metzger, Benson, \& Riccio, 1997; Flint \& Riccio, 1996; Harrod, Metzger, \& Riccio, 1996). Recent results from a place preference study using preweanling rats has also demonstrated that TTW scores are a more sensitive dependent measure than are latency scores (Flint, Bunsey, \& Riccio, 1997a). Neither of the PSEUDO groups appears to be different from the CTRL group, which would suggest that the reactivation treatments did not induce any behavioral side effects that might be construed as good retention. The differences between the CTRL group and the UCS- 40 group hint toward a weak memory reactivation effect. On the other hand, it is clear that the highintensity reactivation treatment elicited a high degree of memory retrieval, resulting in good retention performance for the subjects in this group. This finding suggests that a specific match in the external cues of the training and reactivation stimulus is not likely to be the sole basis of the memory reactivation effect.

An analysis of the test latencies for all the groups, using a Kruskal-Wallis one-way ANOVA, revealed significant differences $(H=17.55, p<.05)$ among the groups. All subsequent pairwise comparisons on test latencies were 


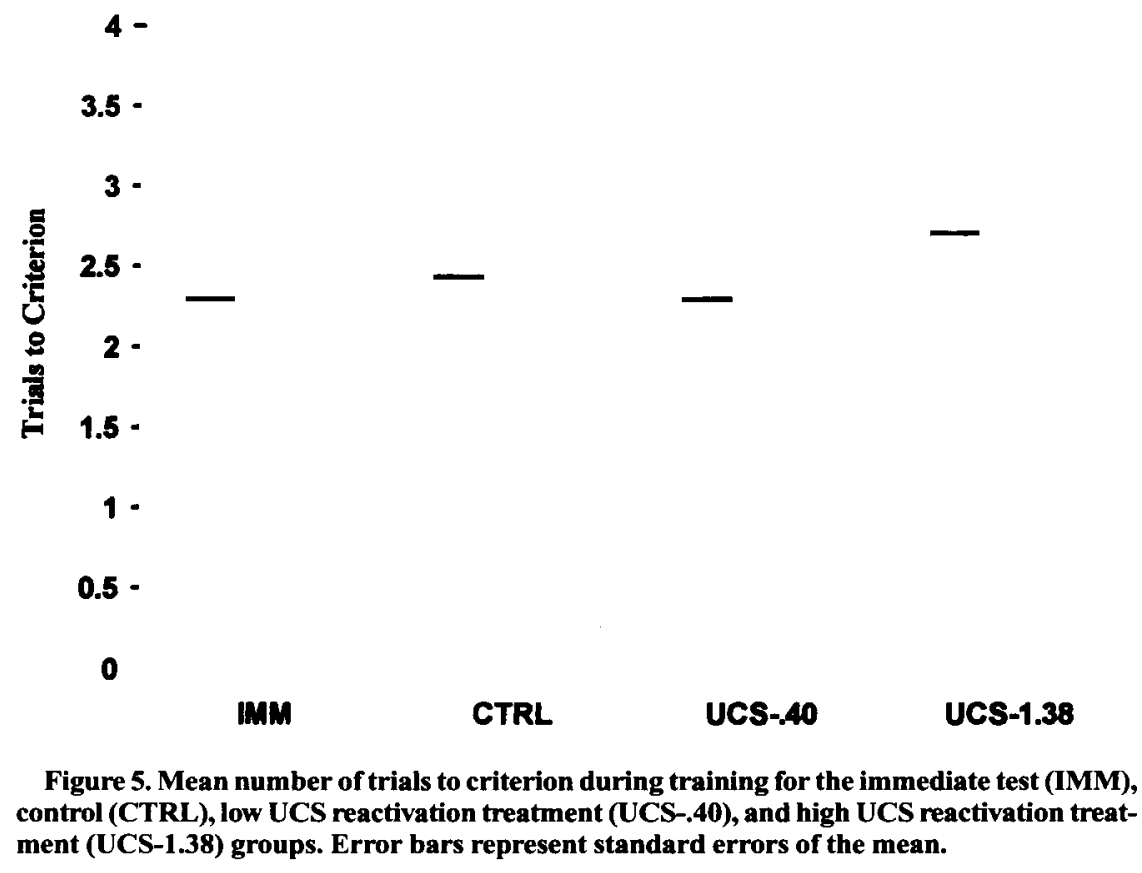

done using the two-tailed Mann-Whitney $U$ test. Similarly, a one-way ANOVA on the TTW scores also indicated that there were differences among the groups $[F(5,44)=$ $15.81, p<.05]$, and all subsequent pairwise comparisons were conducted using Tukey's HSD test. The mean difference $(M D)$ is reported for the Tukey's HSD test.

Comparison between the IMM and the CTRL groups confirmed the previous impressions. The subjects in the IMM group had significantly higher retention scores in comparison with the control animals (latency, $U=0.00$, and TTW, $M D=175.79, p \mathrm{~s}<.01$ ), indicating a rapid rate of forgetting in these immature organisms. An analysis of the pseudo-trained groups in comparison with the controls also confirmed our initial impressions, indicating that neither the PSEUDO-.40 (latency, $U=34.5$, and TTW, $M D=23.16, p \mathrm{~s}>.05$ ) nor the PSEUDO-1.38 (latency, $U=34.00$, and TTW, $M D=46.01, p \mathrm{~s}>.05$ ) group differed from the CTRL group. Despite the potential inclusion of subjects with long crossover latencies in the pseudo-training groups, the lack of difference between these groups and the control group indicate that the reactivation treatments did not have any behavioral side effects that might have been construed as good retention. It is important to note that any subjects in the pseudotrained groups that may have had naturally long crossover latencies would have contributed to an increase in the likelihood of finding a significant difference in comparison with the CTRL group. The fact that no difference existed strengthens the conclusion that the reactivation treatments had no effect on pseudo-trained animals.

The critical comparisons for evaluation of the memory retrieval effects of the UCS reactivation treatment indicated that the CTRL group was not significantly different from the UCS-. 40 group (latency, $U=21.50$, and
TTW, $M D=64.98, p s>.05$ ). This indicates that, although a numerical difference between the group means may exist for the CTRL and the UCS-.40 groups, it does not represent a statistically reliable increase in retention for the reactivation group. On the other hand, the dependent measures for pairwise comparisons between the CTRL and the UCS-1.38 groups were mixed. For latency, a marginally significant difference was found $(U=13.00, p=$ .08 ), suggesting a weak difference on this measure. However, the TTW scores indicated a significant increase in retention scores for the UCS-1.38 group $(M D=141.48$, $p<.001)$.

Additional support for the reactivating effects of pretest footshock comes from the comparisons between the pseudo-trained groups and their corresponding treatments groups. The PSEUDO- 40 group did not differ from the UCS- .40 group on either of the dependent measures (latency, $U=36.00$, and TTW, $M D=18.93, p \mathrm{~s}>.05$ ), whereas the PSEUDO- 1.38 group was significantly different from the UCS-1.38 group on both measures (latency, $U=17.00$, and TTW, $M D=95.43, p \mathrm{~s}<.05$ ). These findings are consistent with the results of the comparisons between the CTRL and the treatment groups.

As was previously indicated, a total of 10 animals were eliminated because they did not cross over during the initial conditioning trial within the allotted time. It is not clear why this occurred in the present experiment and not in Experiment 1. There were no training latencies in the pseudo-training groups, but it is very likely that some of the animals in each group may not have passed the training criterion and, thus, would have been eliminated. As a result of this conditioning procedure, the PSEUDO-.40 and PSEUDO- 1.38 groups had 10 subjects each in order to reduce the impact of any potential outliers. 


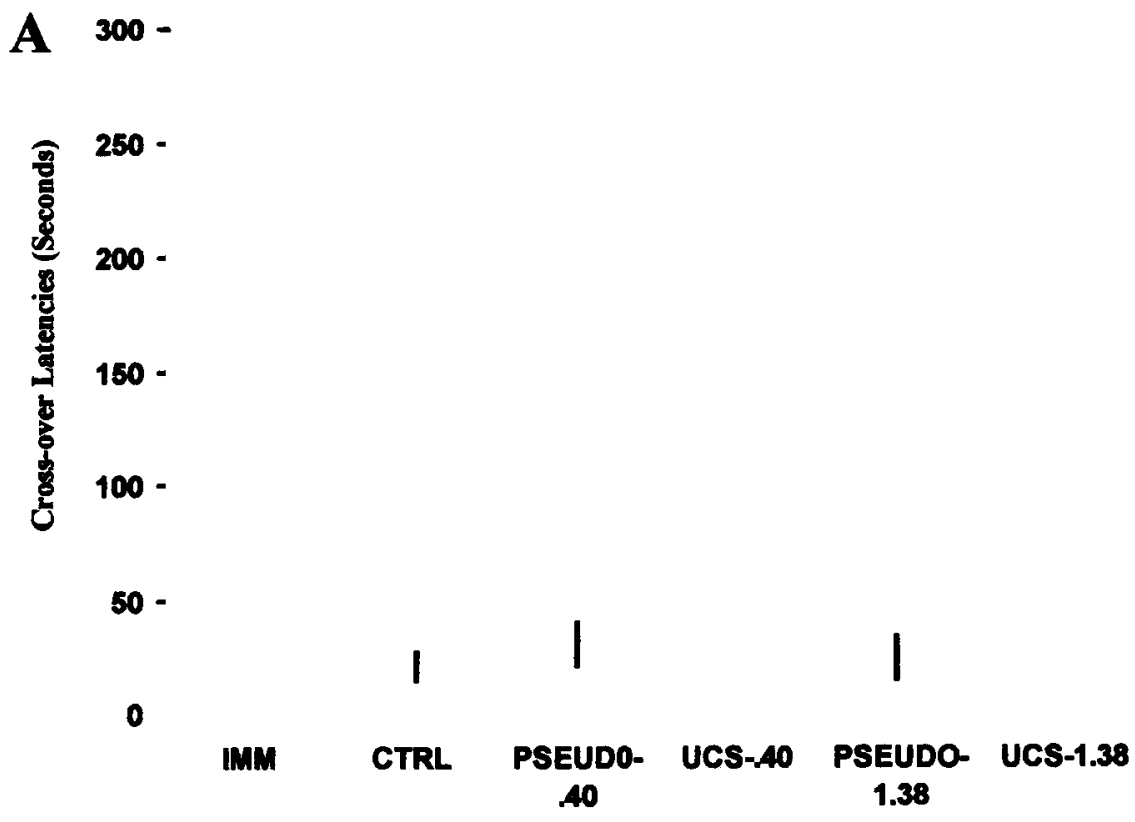

B 300 -

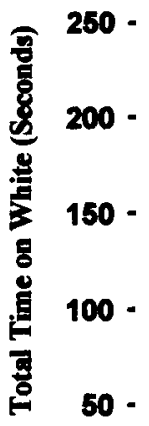

\section{$\mathbf{0}$}

IMI

CTRL

PSEUDO-

UCs-.40

PSEUDO- UCS-1.38

1.38

Figure 6. Mean crossover latencies (panel A) and scores for total time spent on white side (panel B) during testing for the immediate test (IMM), control (CTRL), 0.40-mA pseudo-training (PSEUDO-.40), 1.38-mA pseudo-training (PSEUDO-1.38), low UCS reactivation treatment (UCS-.40), and high UCS reactivation treatment (UCS-1.38) groups. Error bars represent standard errors of the mean.

\section{GENERAL DISCUSSION}

The results of these studies indicate that a match in UCS intensity between the training and the reactivation footshock is not a necessary component in memory reactivation. In both experiments, the low-intensity UCS was suboptimal as a reactivation treatment, particularly in comparison with the high-intensity reactivation treatment. In Experiment 1, the effective reactivation treatment (high UCS intensity) matched the training stimulus, thus replicating previous findings. It is possible that the low-inten- sity footshock reactivation treatment did not serve as an effective reminder because it did not match the training stimulus in terms of intensity and perceptual experience. If the $0.40-\mathrm{mA}$ footshock produced an altogether different stimulus sensation, it would not be surprising that it had no effect on the training memory. However, in Experiment 2 , the intensity of the effective reactivation treatment was more than three times that of the low-intensity stimulus, which actually matched the training stimulus and was ineffective as a reactivation treatment. The outcome of this mismatch between training and reactivation stimuli may 
provide some initial insight into the potentially multifaceted influence of aversive reactivation stimuli.

The most viable alternative explanations involve the activation of stress related neurochemicals, which might serve as internal contextual cues and neuromodulators influencing memory retrieval mechanisms. In support of these possibilities, studies have demonstrated that catecholamines, such as epinephrine, are extremely high immediately following a very high intensity footshock in adult rodents but return to baseline levels within $10 \mathrm{~min}$ following the footshock (McCarty \& Gold, 1981). Although the retrieval test in the present studies occurred approximately $25 \mathrm{~min}$ after the reactivation treatment, other components of the stress cascade related to epinephrine release may be involved. Indeed, it has also been demonstrated that plasma glucose levels increase significantly in response to a high-intensity footshock, but not to a lowintensity footshock, and that the levels remain significantly higher than baseline for at least $30 \mathrm{~min}$ after the footshock (Hall \& Gold, 1986). Similar results on blood glucose levels have been reported following systemic administration of epinephrine (Hall \& Gold, 1986). Because measuring the endogenous levels of plasma epinephrine and glucose was beyond the scope of these studies and the prior research in this area was conducted with adult rodents, these generalizations are made on the basis of the assumption that the neurochemical responses to footshock are similar in preweanling rats.

When considering the alternative neurochemical contributions to the reactivation effect demonstrated with a high-intensity aversive stimulus, it is important to consider that the stress cascade included the alteration of numerous neurochemicals in addition to epinephrine and glucose. It is equally possible that one or all of the neurochemical alterations may play important roles in this retrieval effect (see Gold, 1995, and McGaugh, 1989, for reviews). The site(s) of action of these neuromodulators is not known, but it is believed that they do have a central effect, and strong potential candidates include the adrenergic and/or the cholinergic systems (Dalmaz, IntroiniCollison, \& McGaugh 1993; Durkin, Messier, de Boer, \& Westerink, 1992; McGaugh, 1990; Messier, Durkin, Mrabet, \& Destrade, 1990; Ragozzino, Unick, \& Gold, 1996; Stone, Walser, Gold, \& Gold, 1991).

It is also possible that these neurochemical stress-related responses may have established an internal contextual cue that the organism could have associated with the training episode. In Experiment 2, the animals received multiple trials of weak-intensity passive-avoidance conditioning. These trials may have summated to induce an increase in the release of endogenous chemicals equivalent to that induced by a single high-intensity reactivation treatment. Therefore, the $1.38-\mathrm{mA}$ reactivation treatment may have reestablished an internal cue that served as a contextual reminder of the training episode. One additional possible explanation for the results in Experiment 2 revolves around the rapid rate of forgetting in immature organisms. The low-intensity reactivation treatment may have reactivated the memory for the training episode but could not be maintained during the 25-min delay before the retention test. The results of these studies do not differentiate between these possible explanations, but it is clear that there may be several characteristics of the UCS reminder treatment necessary for memory reactivation.

The passive-avoidance conditioning paradigm employed here has been repeatedly used to demonstrate that preweanling rats, tested $5 \mathrm{~min}$ after training, perform significantly better than those tested $24 \mathrm{~h}$ later (e.g., Flint \& Riccio, 1997, 1998, 1999, 10-min posttraining test). These findings demonstrate the rapid rate of forgetting, often referred to as IA, present in immature, but not in adult organisms. These results are in contrast with those of McKinzie, Chen, and Spear (1998), who recently demonstrated that preweanling rats performed better $24 \mathrm{~h}$ after Pavlovian conditioned emotional response (CER) training than they did 5 min after training. The cause of the differences between these studies is not clear, but it is likely that it revolves around the conditioning criteria employed in the different paradigms. In the present studies, the animals were administered a footshock and immediately returned to the white (safe) compartment, where they were required to demonstrate learned passive avoidance for $180 \mathrm{sec}$. If the subjects entered the black (shock) compartment at any point during this time, they were administered another footshock and returned to the white compartment. This procedure continued until the animals reached the 180 -sec training criterion. In the CER study by McKinzie et al., subjects were exposed to different numbers of CS-US pairings, with variations in the CS, US intensity, retention interval, training session length, housing during the retention interval, and age of the animals. Since the animals were not trained to a particular behavioral criterion, it is possible that they may not have completely consolidated the conditioning episode before the retention test at the 5-min delay (McKinzie et al., 1998). Thus, poorer performance would be demonstrated at a short interval, as opposed to a longer interval. Another explanation might be that the multiple-trial passiveavoidance paradigm provides animals with an extended period of relatively constant exposure to the important contextual cues of the training context. Although this is in contrast to the McKinzie et al. study, in which the CS was presented only briefly, it would seem to be a less feasible explanation of the discrepancies between the paradigms, since it would be difficult to explain the improved performance following a longer delay. These retention differences between the passive-avoidance and CER paradigms in preweanling rats raise important questions regarding animal models of IA and deserve further study.

\section{REFERENCES}

Campbell, B. A., \& Campbell, E. H. (1962). Retention and extinction of learned fear in infant and adult rats. Journal of Comparative \& Physiological Psychology, 55, 1-8. 
Campbell, B. A., \& Jaynes, J. R. (1966). Effect of duration of reinstatement on retention of a visual discrimination learned in infancy. Developmental Psychology, 1, 71-74.

CAMPBell, B. A., JaYNes, J. R., \& Misanin, J. (1968). Retention of a light-dark discrimination in rats of different ages. Journal of Comparative \& Physiological Psychology, 66, 467-472.

Campbell, B. A., \& Spear, N. E. (1972). Ontogeny of memory. Psychological Review, 79, 215-236.

Dalmaz, C., InTroini-Collison, I. B., \& McGaugh, J. L. (1993). Noradrenergic and cholinergic interactions in the amygdala and the modulation of memory storage. Behavioural Brain Research, 58, 167-174.

Durkin, T. P., Messier, C., De Boer, P., \& Westerink, B. H. C. (1992). Raised glucose levels enhance scopolamine-induced acetylcholine overflow from the hippocampus: An in vivo microdialysis study in the rat. Behavioural Brain Research, 49, 181-188.

Flint, R. W., JR., Bunsey, M. D., \& Riccio, D. C. (1997a). [Pre-weanling rat's place preference in a passive-avoidance apparatus]. Unpublished raw data.

Flint, R. W., JR., Bunsey, M. D., \& Riccio, D. C. (1997b). [UCS intensity-dependent vocalizations in preweanling rats]. Unpublished raw data.

Flint, R. W., JR., Metzger, M. M., Benson, D. M., JR., \& Riccio, D. C. (1997). Stress-induced memory enhancement for inhibitory fear conditioning in rats. Psychobiology, 25, 89-94.

Flint, R. W., JR., \& Riccio, D. C. (1996). Glucose administration attenuates hypothermia-induced retrograde amnesia in rats in a timeand dose-dependent manner. Psychobiology, 24, 62-66.

FLINT, R. W., JR., \& Riccio, D. C. (1997). Pre-test administration of glucose attenuates infantile amnesia for passive avoidance in rats. Developmental Psychobiology, 31, 207-216.

FLINT, R. W., JR., \& Riccio, D. C. (1998, May). Dose-dependent modulation of memory retrieval with epinephrine in infant rats. Poster presented at the 70th annual Midwestern Psychological Association Meeting, Chicago.

Flint, R. W., JR., \& Riccio, D. C. (1999). Post-training glucose administration attenuates forgetting of passive-avoidance conditioning in 18-day-old rats. Neurobiology of Learning \& Memory, 72, 62-67.

GoLD, P. E. (1995). Modulation of emotional and nonemotional memories: Same pharmacological systems, different neuroanatomical systems. In J. L. McGaugh, N. M. Weinberger, \& G. Lynch (Eds.), Brain and memory: Modulation and mediation of neuroplasticity (pp. 4174). New York: Oxford University Press.

GoLD, P. E., \& vaN BUSKIRK, R. B. (1975). Facilitation of time-dependent memory processes with posttrial epinephrine injections. Behavioral Biology, 13, 145-153.

GolD, P. E., \& VAN BUSKIRK, R. B. (1976). Enhancement and impairment of memory processes with post-trial injections of adrenocorticotrophic hormone. Behavioral Biology, 16, 387-400.

HALl, J. L., \& Gold, P. E. (1986). The effect of training, epinephrine, and glucose injections on plasma glucose levels in rats. Behavioral Biology, 46, 156-167.

HAROUTUNiAN, V., \& Riccio, D. C. (1977). Effect of arousal conditions during reinstatement treatment upon learned fear in young rats. Developmental Psychobiology, 10, 25-32.

Haroutunian, V., \& Riccio, D. C. (1979). Drug-induced "arousal" and the effectiveness of CS exposure in the reinstatement of memory. Behavioral \& Neural Biology, 26, 115-120.

Harrod, S. B., MetzGer, M. M., \& Riccio, D. C. (1996). Does induced recovery from amnesia represent a disinhibition effect? Physiology \& Behavior, 60, 1375-1378.

Holson, R. R., \& Pearce, B. (1992). Principles and pitfalls in the analysis of prenatal treatment effects in multiparous species. Neurotoxicology \& Teratology, 14, 221-228.

Howe, M. L., \& CouRage, M. L. (1993). On resolving the enigma of infantile amnesia. Psychological Bulletin, 113, 305-326.

KIRBY, R. H. (1963). Acquisition, extinction, and retention of an avoid- ance response in rats as a function of age. Journal of Comparative \& Physiological Psychology, 56, 158-162.

MCCARTY, R., \& Gold, P. E. (1981). Plasma catecholamines: Effects of footshock level and hormonal modulators of memory storage. Hormones \& Behavior, 15, 168-182.

MCGAUGH, J. L. (1989). Involvement of hormonal and neuromodulatory systems in the regulation of memory storage. Annual Review of Neuroscience, 12, 255-287.

MCGAUGH, J. L. (1990). Significance and remembrance: The role of neuromodulatory systems. Psychological Science, 1, 15-25.

McKinzie, D. L., Chen, W. J. A., \& SPEAR, N. E. (1998). Ontogenetic differences in the expression of conditioned stimulus conditioning: Effects of retention interval. Behavioral Neuroscience, 112, 920-928.

Messier, C., Durkin, T., Mrabet, O., \& Destrade, C. (1990). Memoryimproving action of glucose: Indirect evidence for a facilitation of hippocampal acetylcholine synthesis. Behavioural Brain Research, 39, 135-143.

Miller, J. S., Jagielo, J. A., \& Spear, N. E. (1991). Differential effectiveness of various prior-cuing treatments in the reactivation and maintenance of memory. Journal of Experimental Psychology: Animal Behavior Processes, 17, 249-258.

Ragozzino, M. E., UNick, K. E., \& Gold, P. E. (1996). Hippocampal acetylcholine release during memory testing in rats: Augmentation by glucose. Proceedings of the National Academy of Sciences, 93, 4693-4698.

Riccio, D. C., Rohrbaugh, M., \& Hodges, L. A. (1968). Developmental aspects of passive and active avoidance learning in rats. $D e$ velopmental Psychobiology, 1, 108-111.

RoozendaAl, B., Williams, C. L., \& MCGaugh, J. L. (1999). Glucocorticoid receptor activation in the rat nucleus of the solitary tract facilitates memory consolidation: Involvement of the basolateral amygdala. European Journal of Neuroscience, 11, 1317-1323.

RoveE-COllier, C., \& HAYNe, H. (1987). Reactivation of infant memory: Implications for cognitive development. In H. W. Reese (Ed.), Advances in child development and behavior (Vol. 20, pp. 185-238). San Diego: Academic Press.

Rovee-Collier, C., \& Shyi, G. (1992). A functional and cognitive analysis of infant long-term retention. In M. L. Howe, C. J. Brainerd, \& V. F. Reyna (Eds.), Development of long-term retention (pp. 3-55). New York: Springer-Verlag.

SANDI, C. (1998). The role and mechanisms of action of glucocorticoid involvement in memory storage. Neural Plasticity, 6, 41-52.

SPEAR, N. E., \& Parsons, P. J. (1976). Analysis of a reactivation treatment: Ontogenetic determinants of alleviated forgetting. In D. L. Medin, W. A. Roberts, \& R. T. Davis (Eds.), Processes of animal memory (pp. 135-165). Hillsdale, NJ: Erlbaum.

SPEAR, N. E., \& RICCio, D. C. (1994). Memory: Phenomena and principles. Boston: Allyn \& Bacon.

Stone, W. S., Walser, B., Gold, S. D., \& Gold, P. E. (1991). Scopolamine- and morphine-induced impairments of spontaneous alternation performance in mice: Reversal with glucose and with cholinergic and adrenergic agonists. Behavioral Neuroscience, 105, 264-271.

USHER, J. A., \& NeISSER, U. (1993). Childhood amnesia and the beginnings of memory for four early life events. Journal of Experimental Psychology: General, 122, 155-165.

White, N. (1991). Peripheral and central memory-enhancing actions of glucose. In R. C. A. Frederickson, J. L. McGaugh, \& D. L. Felten (Eds.), Peripheral signaling of the brain: Role in neural-immune interactions and learning and memory (pp. 421-441). Lewiston, NY: Hogrefe \& Huber.

WhITE, N., \& Legree, P. (1984). Effect of post-training exposure to an aversive stimulus on retention. Physiological Psychology, 12, 233236.

(Manuscript received May 19, 1999; revision accepted for publication July 20, 1999.) 